\title{
REDES DE IGUALES Y ACOSO ESCOLAR: EVALUACIÓN DESDE EL ANÁLISIS DE REDES SOCIALES
}

\author{
PEER NETWORKS AND BULLYING: AN EVALUATION FROM THE SOCIAL NETWORK ANALYSIS
}

\author{
José HeRnANDo ÁvILA-TOSCANO*, AILED DANIELA MARENCO-ESCUDEROS**, \\ MARÍA ANGÉLICA TILANO OSORIO*** \\ CORPORACIÓN UNIVERSITARIA REFORMADA, BARRANQUILLA - COLOMBIA
}

FECHA RECEPCIÓN: 10/12/2012 • FECHA ACEPTACIÓN: 14/02/2013

\begin{abstract}
Resumen
El objetivo de este estudio consistió en identificar las características de las redes sociales de 320 estudiantes de secundaria implicados en conductas de acoso, con el fin de observar posibles diferencias en la estructura de sus relaciones de acuerdo a los roles asumidos (observador, agresor, víctima, agresor-víctima). Mediante un diseño ex post facto se analizaron las propiedades de las redes de acuerdo a los indicadores de centralidad de grado, cercanía e intermediación; la comparación de estas propiedades se cumplió mediante pruebas no paramétricas de diferencias entre grupos. Los resultados indican rendimientos similares de las características de las redes indistintamente del rol asumido; se observó un número amplio de agresores-víctimas como actores centrales en las redes al igual que víctimas con posiciones sociales importantes.
\end{abstract}

Palabras clave: redes sociales, intimidación, jóvenes.

\section{Abstract}

The aim of this study was to identify the characteristics of social networks of 320 high school students involved in bullying behaviors, in order to observe possible differences in the structure of their relationships according to the roles assumed (observer, aggressor, victim, aggressorvictim). Using an ex post facto design analyzed the properties of the networks according to indicators of degree, closeness and betweenness, the comparison of these properties are fulfilled with nonparametric tests of differences between groups. The results indicate similar performance of the network characteristics regardless of the role assumed, there was a large number of aggressor-victim as central actors in networks like victims with important social positions.

Keywords: social networks, bullying, youth.

\section{Introducción}

Las relaciones sociales entre pares es un tema primario de investigación en psicología aplicada, su estudio permite ampliar tanto la comprensión de las dinámicas de interacción de los jóvenes como la aproximación a sus problemáticas y desviaciones. En la actualidad ha crecido el interés por el estudio de las redes sociales coligadas al acoso escolar, con el fin de hallar en los patrones de inte-

* Dirección de Investigaciones. Vicerrectoría Académica y de Investigaciones. Grupo de Investigación Psicología, Cultura y Sociedad (PSICUS). Carrera 38 n. ${ }^{\circ}$ 74-179 (Barranquilla, Colombia). Correspondencia: javila@unireformada.edu.co

** Facultad de Ciencias Sociales, Artes y Humanas. Programa de Psicología. Investigadoras adscritas al Grupo de Investigación Psicología, Cultura y Sociedad (PSICUS). 
racción cotidiana mejores y más complejas explicaciones a las prácticas de acoso o intimidación entre pares.

El reconocimiento de la importancia de las redes frente al acoso entre pares (bullying) implica la comprensión de la red como una estructura social que se construye con base a un sentimiento de identidad, de manera que la pertenencia a los círculos de iguales se asocia a una experiencia de compañía que lleva a sentir mayor impulso por la organización y participación (Madariaga, Gallardo, Salas \& Santamaría, 2001), aumentando además la identificación que los individuos construyen con otras personas así como la satisfacción general con el contexto y con la vida (Bidart \& Degenne, 2005). La red surte a los individuos de recursos sociales de apoyo (Ávila-Toscano, 2009; Ávila-Toscano \& Madariaga, 2012) y la comunicación dada mediante la expresión verbal y no verbal dentro de sus dinámicas de relación permite la transmisión de signos emocionales que representan un mínimo de contacto social (Takács, 2007). Sin embargo, las redes no cumplen exclusivamente con funciones positivas sino que sus efectos pueden ser adversos. La interacción también implica relaciones de poder e intercambios desiguales (Samper, 2004), de hecho, en muchas ocasiones el orden social interno conlleva a la privación de recursos, la exclusión y la estigmatización (Carter \& Feld, 2004), convirtiendo las redes de los afectados en sistemas desmedidamente pequeños (Bloom \& Kessler, 1994).

En los centros educativos las redes sociales están condicionadas por la conformación de salones de clases, sin embargo, la escogencia de los actores con quienes se comparte a diario es una tarea que obedece a intereses particulares y a las diferencias individuales de cada actor (Kalish \& Robins, 2006). Los interlocutores sociales se seleccionan con base a las relaciones de influencia que se establecen en el contacto entre pares, puesto que la red no es una construcción individualista sino que su dinámica implica la concepción del grupo; en este proceso actúan tanto los compañeros cercanos como aquellos que ejercen conductas de acoso.

La estructura de las redes de iguales puede ser un escenario de tensiones interpersonales (Ortega, 2002) en donde las prácticas del maltrato constituyen un fenómeno de dominio-sumisión (Avilés \& Monjas, 2005) que sistemática y repetidamente implica el desarrollo de acoso, maltratos, abusos físicos, psicológicos y exclusión social entre compañeros (Cepeda-Cuervo, Pacheco-Durán, García-Barco \& Piraquive-Peña, 2008; Del Barrio et ál., 2008; Garaigordobil \& Oñederra, 2008; Plata, Riveros \& Moreno, 2010), cuya dinámica encuentra en la escuela un núcleo primordial para su desarrollo al ser una comunidad de pares que comparten a diario experiencias de socialización e intercambios de recursos.

Por su parte, los menores asumen de forma diferente la experiencia de maltrato de acuerdo al rol que desempeñan (agresor, víctima, testigo, agresor-víctima) y a otras características como el sexo, la edad y el nivel de desarrollo cognitivo y moral (Hoyos, Aparicio, Heilbron \& Schamun, 2004); por ejemplo, en general se ha observado entre los estudiantes victimizados experimentan sentimientos intensos de vergüenza e inferioridad (Zabaraín \& Sánchez, 2009), mientras que los agresores con facilidad reconocen que atacan a sus compañeros para conseguir un mayor nivel de poder dentro de las relaciones sociales (Kulig, Hall \& Kalischuk, 2008).

Ahora bien, la evidencia empírica resalta los efectos que directa o indirectamente generan los episodios de acoso sobre el desarrollo psicológico y la salud mental de los implicados. Al tratarse de un fenómeno repetitivo, insidioso y en el que además los estudiantes ven afectados su medios sociales de apoyo, resulta común el registro de afectaciones psicológicas a mediano y largo plazo que constituyen problemáticas de comportamiento. Algunos estudios señalan que la experiencia de rechazo desde los primeros años de formación puede conducir al fortalecimiento de conductas reactivas entre los menores disponiéndolos para la agresión (Boivin, 2005), así mismo, entre agresores y víctimas suelen ser comunes desde problemas psicosomáticos (Gini \& Pozzoli 2009), hasta conductas que revisten mayor gravedad como las autolesiones, el comportamiento violento y la sintomatología psicótica (Arseneault, Bowes \& Shakoor, 2010), llegando a la concepción de ideación suicida (Hinduja \& Patchin, 2010).

Este tipo de problemáticas pueden ser potenciadas por los efectos de los patrones de influencia dentro de las redes sociales, en especial cuando el número y la calidad en las amistades (Gini \& Pozzoli 2009), en los menores es deficiente, lo que disminuye la posibilidad de acceder a recursos de apoyo que permitan paliar o mitigar los efectos negativos del maltrato, cuya práctica 
se naturaliza dentro de las relaciones entre pares en la escuela.

Precisamente, una de las principales problemáticas asociadas al bullying es que suele ser asumido como una forma legítima de obtención de posición social y de instauración de orden en las relaciones (Riaño, 2008), llegando a mediar en el nivel de influencia social que poseen los menores en la red. Algunas investigaciones han reportado a los agresores como individuos muy integrados a los grupos sociales (Farmer et ál., 2010; Witvliet et ál., 2010), y con tendencia a figurar como los lideres de estos, mientras que las víctimas suelen ser sistemáticamente rechazadas ubicándolas al margen de la red (Estell, Farmer \& Cairns, 2007). Otros hallazgos señalan que en general agresores y víctimas experimentan diferentes niveles de inadaptación social, pero en el caso de los estudiantes maltratados esta condición es mucho más acentuada pues suelen ser más pobres en el ajuste emocional y social, presentan peores relaciones con sus compañeros, experimentan un mayor sentimiento de soledad y tienen más dificultades para hacer amigos (Nansel et ál., 2001). Esta información coincide con las descripciones de Cerezo y Ato (2010), quienes identifican a las víctimas como un grupo compacto que frecuentemente es rechazado y excluido de los juegos y demás actividades, lo que a su vez define un bajo nivel de relaciones interpersonales para este grupo de niños dentro de la escuela.

La mayor influencia social de los agresores y la desproporcionada reducción de la red de las víctimas también ha sido reportada por Xie, Farmer y Cairns (2003), tanto en adolescentes hombres como en mujeres; los autores hallaron que los varones que actúan con agresiones físicas o verbales y las chicas que ejercen agresiones de tipo relacional tienen niveles más altos de centralidad en la red social escolar que los compañeros que no participan de las hostilidades.

Al parecer, la adopción de una conducta hostil y la aceptación de la misma se relaciona con la sensación de pertenencia a un subgrupo dentro de la red, Ojala y Nesdale (2004) identificaron que el bullying es más aceptable cuando el daño se dirige hacia un miembro de un grupo diferente que sea percibido como una posible amenaza al grupo de pertenencia, así mismo, el comportamiento de un miembro depende en gran medida del patrón de conducta que sea aceptado por el grupo, de forma que si se da una conducta hostil, el grupo consiente este tipo de socialización.

Frente a este panorama, el Análisis de Redes Sociales (ARS) cobra un papel protagónico en las investigaciones sobre bullying, pues permite obtener información sobre la red de amistad y la influencia de la misma en las situaciones de acoso (Merino, 2008). El ARS es un método cuantitativo basado en la identificación de la regularidad de relaciones establecidas por actores sociales (Hawe, Webster \& Shiell, 2004), las cuales están influidas por las condiciones normativas de la red, de forma que se asume que las características de las relaciones determinan el comportamiento de los individuos que forman parte de dicha red (Trujillo, Mañas \& González-Cabrera, 2010). Desde esta perspectiva, lo social se constituye mediante el establecimiento de vínculos entre actores y no se interpreta exclusivamente desde la identificación de atributos individuales (Hawe et ál., 2004); esta metodología favorece el estudio de la influencia social en las relaciones de acoso pues el modelo de ARS cuenta con un diseño que ayuda a comprender la dinámica social en la escuela, y a la vez permite emplearlo como un instrumento preventivo para la detección de las condiciones sociales en el medio educativo que exponen a las prácticas de maltrato y exclusión (Merino, 2008).

Precisamente, en consideración de la importancia de analizar el fenómeno del acoso y el papel de las redes de interacción, este estudio se ha planteado dos objetivos fundamentales:

1. Identificar las posibles manifestaciones de acoso entre iguales de una comunidad educativa diferenciando los roles asumidos por los estudiantes, así como la relación de dichos roles con el sexo y el nivel de formación.

2. Establecer si existen diferencias en las características estructurales de las redes sociales de acuerdo al sexo y a los roles asumidos por los estudiantes en las situaciones de acoso.

A través del desarrollo de estos objetivos, se responde a una necesidad investigativa que contempla el papel de la estructura de relaciones sobre una problemática psicosocial y de salud psicológica como lo es el maltrato o acoso entre iguales, de esta forma se asume -en congruencia con la línea de razonamiento de Gifford-Smith y Brownell (2003- que las interacciones 
ocurridas en los grupos de compañeros afectan el desarrollo y funcionamiento psicoemocional, la capacidad de adaptación psicosocial, y probablemente también todos los demás aspectos de la vida (incluyendo la familia, la escuela y la comunidad), lo que hace indispensable su análisis.

\section{Método}

\section{Diseño}

De acuerdo con la clasificación de Montero y León (2007), el estudio desarrollado consistió en un diseño ex post facto, de un grupo, simple. En el cual se seleccionó un conjunto de estudiantes naturalmente constituidos en redes de iguales en las que se evaluó la existencia de manifestaciones de acoso escolar, con el fin de analizar las propiedades estructurales de dichas redes en función del tipo de participación en las conductas de acoso.

\section{Participantes}

En el estudio se incluyó un total de 320 estudiantes divididos en cinco grupos académicos de educación secundaria obligatoria ubicados en grado octavo $(n=162 ; 50.6 \%)$ y noveno $(n=158 ; 49.4 \%)$, los cuales pertenecían a una institución educativa privada de la ciudad de Barranquilla (Costa Caribe, Colombia, Suramérica). Los participantes contaban con edades comprendidas entre los 12 y los 16 años $(M=13.92$; $D E=.783), 192$ fueron hombres (60\%) y 128 mujeres (40\%). En relación con la extracción socioeconómica se observó que la mayoría de los participantes pertenecían a un nivel medio (estrato $3: n=86 ; 26.9 \%$ y estrato $4: n=153 ; 47.8 \%$ ), seguido de un número importante de estudiantes de estrato social alto (estrato 5 : $n=54 ; 16.9 \%$ y estrato $6: n=14 ; 4.4 \%$ ); finalmente, $4.1 \%(n=13)$ pertenecía a una condición económica baja (estrato 2).

\section{Instrumentos}

Las conductas de acoso se evaluaron con la versión actualizada del Cuestionario del Defensor del Pueblo (2007), cuyo diseño se ha basado en la revisión de un amplio número de investigaciones a nivel internacional, a partir de las cuales se han desarrollado adaptaciones específicas que han permitido una medición confiable y precisa. El instrumento consta de tres secciones principales (acoso observado, realizado y experimentado) en las que se identifican los tipos de maltrato y sus formas de presentación desde la perspectiva de los diversos actores implicados. Adicionalmente, incluye una sección para la identificación de información demográfica de los participantes (edad, curso, género), así como una sección para identificar escenarios en los que se presentan las conductas de maltrato. El cuestionario se responde mediante la selección de reactivos distribuidos en escala tipo Likert con respuesta del tipo Nunca, A veces, A menudo y Siempre, y asume que las respuestas se basan en el reporte de ocurrencia continuada, por lo cual la intensidad de los episodios de acoso se entienden como frecuencia de casos en que se experimenta el acoso.

La evaluación de las redes se cumplió mediante la aplicación de un instrumento consistente en una matriz cuadrada de datos reticulares, diseñada para el cruce de información basada en el reporte de afiliaciones o vinculaciones entre los diferentes actores de cada grupo estudiado, siguiendo la metodología ejecutada en estudios previos (Ávila-Toscano, Gutiérrez \& Pérez, 2011). Este instrumento permite identificar las relaciones existentes entre los miembros de la red definiendo el nivel de vinculación o separación en las relaciones, lo cual facilita la obtención de indicadores estructurales que definen las propiedades cuantitativas de las redes.

\section{Procedimiento}

El instrumento para la medición de redes se adaptó a las condiciones de la muestra mediante una prueba piloto con 63 estudiantes de ambos sexos, con edades y nivel de formación similares a los de la muestra. Tras la corrección semántica y el ajuste de la prueba se procedió al proceso de información de los propósitos del estudio a los directivos de la institución educativa seleccionada, y una vez obtenida la aprobación institucional se realizó el proceso de consentimiento informado tanto de los acudientes como de los estudiantes de los cursos seleccionados, a quienes se les explicó los lineamientos investigativos y posteriormente se firmó el consentimiento bajo los principios de vinculación voluntaria, respeto por la intimidad y ausencia de riesgo psicológico, moral o social. 
Los grupos fueron seleccionados con base en los criterios de Avilés y Monjas (2005) entre los grados octavo y noveno en el año escolar, sin hacer distinción de género, rendimiento intelectual, ni antecedentes de conducta conflictiva en la escuela con el fin de lograr grupos naturalmente constituidos y heterogéneos.

Inicialmente se realizó la medición del acoso escolar-bullying de forma individual y autoaplicada y posteriormente, bajo las mismas condiciones se evaluó la red social. El orden de aplicación de los instrumentos se controló mediante constancia y el proceso de cumplimiento de los cuestionarios contó con la supervisión de los investigadores que estuvieron dispuestos a orientar a los estudiantes que así lo requirieron.

\section{Análisis de datos}

El análisis de los datos se realizó en dos fases de acuerdo a los objetivos propuestos. Inicialmente (fase 1), se cumplió con un procedimiento de análisis descriptivo para la identificación de las principales manifestaciones de maltrato y de los roles asumidos dentro de las relaciones de acoso por los participantes, a estos últimos se les categorizó en un único rol, evitando que se sumaran en los roles restantes, siguiendo criterios aplicados en estudios previos (Ávila-Toscano, Osorio, Cuello, Cogollo \& Causado, 2010) y definiendo cuatro roles esenciales: $o b$ servador (estudiantes no victimizados ni agresores), víctima (participantes que reportaban la observación de maltrato y que al tiempo sufrían episodios de bullying), agresor (estudiantes que ejercían acoso y que no eran objeto de hostilidades) y agresor-víctima (estudiantes que ejercían conductas de acoso y que a la vez eran objeto de agresiones).

Así mismo, se aplicó el Chi cuadrado de Pearson $\left(x^{2}\right)$ para evaluar la existencia de relaciones entre el rol asumido, el sexo y nivel de formación de los participantes.

Posteriormente (fase 2), se analizaron las propiedades estructurales de las redes mediante el software Ucinet 6,391 (Borgatti, Everett \& Freeman, 2002), a través del cual se calcularon indicadores que miden la centralidad y el poder social que, para el caso del estudio, se basó en cuatro indicadores esenciales definidos a continuación:

-> Grado nodal. Nivel de participación social y control de relaciones definidos por el número de vínculos establecidos en la red.
-> Cercanía de entrada. Define la distancia en que los demás identifican a un actor determinado.

$\longrightarrow$ Cercanía de salida. Define la distancia en que un actor determinado identifica a los demás.

$\longrightarrow$ Intermediación. Medida en que un actor actúa de intermediario en las relaciones de otros dos actores.

La identificación de estos indicadores o propiedades estructurales permite conocer las posiciones sociales asumidas por los individuos y el nivel de control social de las relaciones. Además de ello se construyeron grafos de redes empleando el programa NetDraw 2.119 (Borgatti, 2002), que permite una visualización del nivel de complejidad en las relaciones. Finalmente, los resultados de los indicadores estructurales fueron comparados de acuerdo al rol de cada participante con el SPSS 18.0 mediante la aplicación de estadísticos no paramétricos.

\section{Resultados}

\section{Evaluación de conductas de acoso y roles asumidos por los participantes}

La evaluación de las hostilidades existentes entre los participantes aparece en la Tabla 1, de acuerdo a las tres perspectivas esenciales, la de los testigos, los individuos victimizados y los agresores. Las diferencias frente a los reportes es notoriamente variable desde cada perspectiva, saltando a la vista que son las personas que observan las situaciones de acoso, quienes reconocen un mayor porcentaje de desarrollo de esta clase de comportamientos, mientras que entre agresores y victimizados el reporte es inferior en frecuencia.

Curiosamente, quienes realizan conductas como no dejar participar, insultar, y usar sobrenombres ofensivos, reportan las mismas en mayor porcentaje que quienes las han experimentado, de igual forma, el margen de reporte de acciones hostiles graves como la amenaza y el acoso de tipo sexual es bastante bajo en comparación a la opinión de los testigos, quienes expresan una prevalencia de tales conductas en $56.6 \%$ y $45.6 \%$ respectivamente.

En general prevalecen las conductas hostiles basadas en agresiones de tipo psicológico y relacional (ignorar, hablar mal de los demás, impedir la participación social), seguidas de las agresiones físicas indirectas (robar, destruir y esconder cosas). 
Tabla 1. Reporte general de conductas de acoso en la muestra.

\begin{tabular}{lccc}
\hline \hline Identificación de conductas de acoso & & & \\
\hline Conducta & Observado & Realizado & Experimentado \\
\hline Ignorar & $71.3 \%$ & $50.3 \%$ & $27.8 \%$ \\
No dejar participar & $74.7 \%$ & $22.8 \%$ & $23.4 \%$ \\
Insultar & $70.3 \%$ & $55.0 \%$ & $41.9 \%$ \\
Poner sobrenombres & $72.2 \%$ & $55.3 \%$ & $43.8 \%$ \\
Hablar mal & $81.6 \%$ & $28.1 \%$ & $34.7 \%$ \\
Esconder las cosas & $75.0 \%$ & $38.1 \%$ & $35.3 \%$ \\
Romper las cosas & $74.4 \%$ & $7.2 \%$ & $18.8 \%$ \\
Robar & $65.0 \%$ & $9.7 \%$ & $18.8 \%$ \\
Pegar & $80.6 \%$ & $24.7 \%$ & $22.5 \%$ \\
Amenazar & $56.6 \%$ & $3.8 \%$ & $5.5 \%$ \\
Acosar sexualmente & $45.6 \%$ & $2.2 \%$ & $4.7 \%$ \\
\hline \hline
\end{tabular}

Por su parte, el reporte de los roles asumidos frente a estos comportamientos (Tabla 2) permitió identificar que $18.8 \%(n=60)$ de los participantes manifestó el rol de víctima, mientras que 23.4\% $(n=75)$ el rol de observador; $21.6 \%(n=69)$ respondió al rol de agresor, y finalmente la mayoría de los participantes fueron identificados con el rol mixto de agresores-víctima con 36.3\% $(n=116)$.

Tabla 2. Distribución de los participantes según sexo y curso de acuerdo al rol asumido en las conductas de acoso.

\begin{tabular}{llccrl}
\hline \multirow{2}{*}{ Rol del actor } & \multirow{2}{*}{ Distribución } & \multicolumn{2}{c}{ Sexo } & \multicolumn{2}{c}{ Escolaridad } \\
\cline { 3 - 6 } & & Hombre & Mujer & Octavo & Noveno \\
\hline Observador & $23.4 \%(\mathrm{n}=75)$ & $44 \%(\mathrm{n}=33)$ & $56 \%(\mathrm{n}=42)$ & $25.9 \%(\mathrm{n}=42)$ & $20.9 \%(\mathrm{n}=33)$ \\
Víctima & $18.8 \%(\mathrm{n}=60)$ & $60 \%(\mathrm{n}=36)$ & $40 \%(\mathrm{n}=24)$ & $20.4 \%(\mathrm{n}=33)$ & $17.1 \%(\mathrm{n}=27)$ \\
Agresor & $21.6 \%(\mathrm{n}=69)$ & $58 \%(\mathrm{n}=40)$ & $42 \%(\mathrm{n}=29)$ & $19.1 \%(\mathrm{n}=31)$ & $24.1 \%(\mathrm{n}=38)$ \\
Agresor-Víctima & $36.3 \%(\mathrm{n}=116)$ & $71.6 \%(\mathrm{n}=83)$ & $28.4 \%(\mathrm{n}=33)$ & $34.6 \%(\mathrm{n}=56)$ & $38 \%(\mathrm{n}=60)$ \\
\hline Total & $100 \%(\mathrm{n}=320)$ & $60 \%(\mathrm{n}=192)$ & $40 \%(\mathrm{n}=128)$ & $50.6 \%(\mathrm{n}=162)$ & $49.4 \%(\mathrm{n}=158)$ \\
\hline
\end{tabular}

Los roles asumidos mostraron relación estadísticamente significativa con el sexo de los participantes $\left(x^{2}[3]=14.568 ; p=.002<.01\right)$, siendo en su mayoría hombres quienes asumieron los roles de agresor (58\%), víctima (60\%) y agresor-víctima (71.6\%), contrastando con la distribución de las mujeres quienes se identificaron principalmente con el papel de observadoras (56\%). Entre tanto, la distribución de los roles asumidos de acuerdo al nivel de formación de los estudiantes permitió identificar porcentajes muy similares entre ambos cursos analizados sin que se observaran relaciones significativas a nivel estadístico $\left(x^{2}[3]=2.478\right.$; $p=.479>.05)$.

\section{Características de las redes sociales y comparación de propiedades según rol y sexo}

Una vez definidos los patrones de acoso y con la identificación de los roles desempeñados por cada participante, se procedió a evaluar las propiedades estructurales de las redes de socialización, mediante la medición 
de variables cuantitativas que definen su estructura y la centralidad social.

Los resultados de las propiedades estructurales de las redes demostraron valores diversos. Para el caso del grado nodal de entrada la media obtenida señaló un nivel elevado de interacciones reportadas desde la red hacia cada actor $\left(M=80.21 ; M_{i n}=38.5, M_{d x}=100 ; D E=53.1\right)$, coincidiendo con un reporte también alto de interacciones en sentido inverso, es decir, contactos directos de cada actor hacia el resto de la red o grado nodal de salida $(M=77.6 ; D E=20.98)$, aunque en este caso los valores extremos fueron mucho más diversos $\left(M_{i n}=.00\right.$, $\left.M_{a x}=100\right)$ señalando estudiantes que no reportan contacto alguno con sus redes (muy aislados) y otros con capacidad de interactuar directamente con todos sus compañeros.

La cercanía de entrada obtuvo una media de $79.20\left(D E=14.04 ; M_{\text {in }}=40.29, M_{a x}=100\right)$, mientras que el valor de salida para este indicador fue de 82.77 $\left(D E=14.42 ; M_{i n}=3.57, M_{d x}=100\right)$, lo cual también señala un rendimiento importante en materia de percep- ción de proximidad en el contacto con los pares. Contrariamente, para el caso de la intermediación los valores obtenidos fueron reducidos $\left(M=.65 ; D E=.48 ; M_{\text {in }}=.00\right.$, $\left.M_{\text {dx }}=2.83\right)$, si bien esto puede deberse a que los otros indicadores señalan un nivel elevado de contactos directos, por lo cual el uso de intermediarios es menos frecuente.

Las figuras $1 \mathrm{a}$ y $1 \mathrm{~b}$ ofrecen una visualización detallada de la forma como estas propiedades estructurales se traducen en patrones de relación entre los actores de las redes. En el primer caso, se observa una red general del grado octavo (cuadrante izquierdo) en el que la mayoría de los participantes gozan de posiciones centrales dentro del flujo relacional, aún quienes poseen menor centralidad cuentan con un buen nivel de interacciones. Así mismo, se puede identificar que el intercambio relacional de acuerdo al rol de los actores es muy semejante (cuadrante derecho), sobresale un número importante de agresores y agresores-víctimas que expresan relaciones bidireccionales con todos los demás compañeros. En este caso las víctimas son pocas ( 3 actores) pero gozan de buenas posiciones sociales.

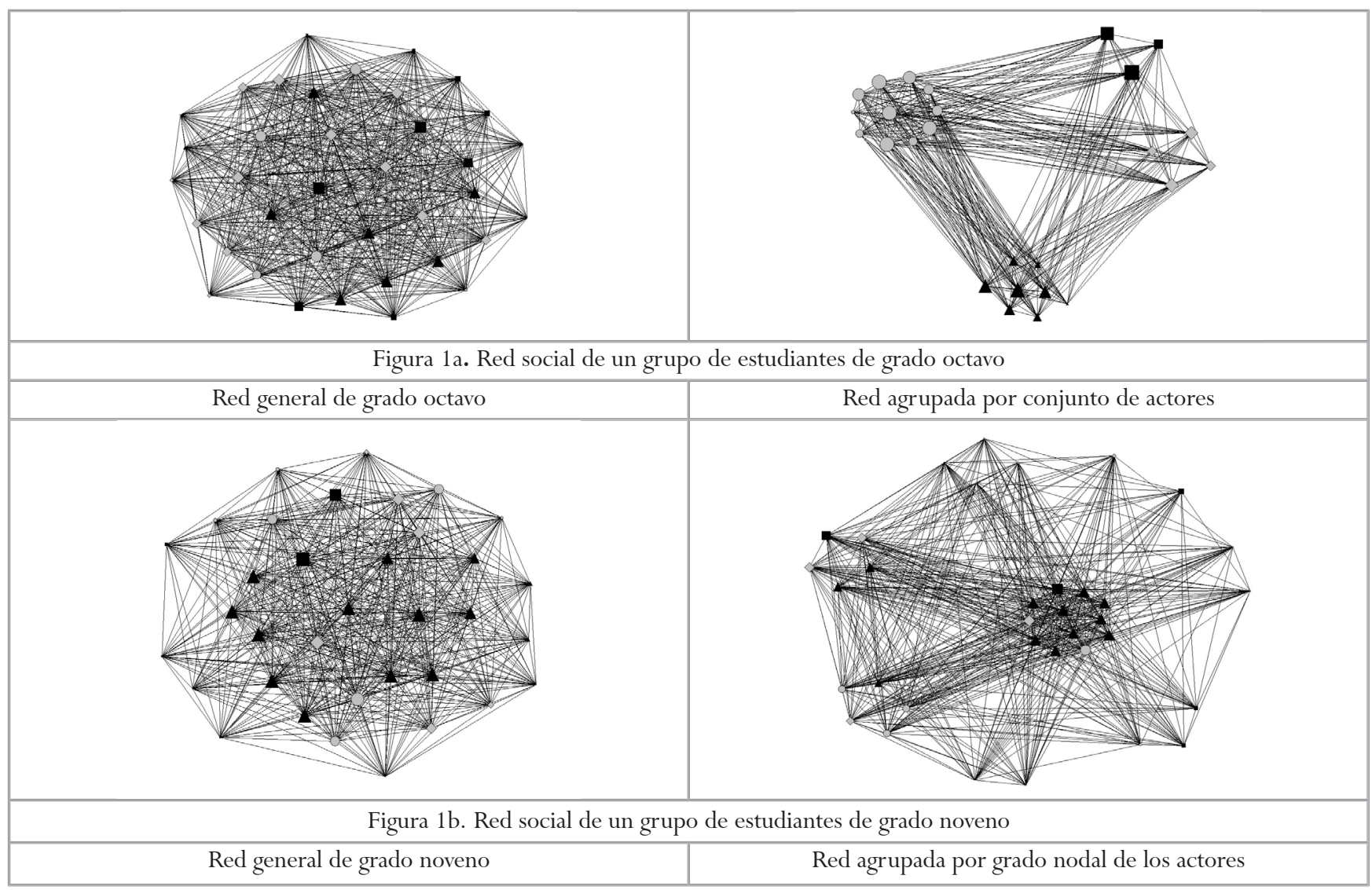

Nota: Círculo: observador. Cuadrado: víctima. Rombo: agresor. Triángulo: agresor-víctima. Tamaño: centralidad dentro de la red. 
La figura $1 \mathrm{~b}$ (cuadrante izquierdo) visualiza una red de grado noveno con mayor número de actores pero con propiedades similares a las descritas previamente, en este caso, es llamativo un número elevado de agresoresvíctimas cuya posición social es sobresaliente (cuadrante derecho), siendo el grupo con más facilidad de interacción con el resto de los contactos independientemente del rol que los mismos asuman.

Con el fin de comprobar si las propiedades de las redes mostraban diferencias significativas según el tipo de participante, se realizó un análisis de diferencias entre grupos definidos por el rol y por el sexo, al haber resulta- do significativa esta última variable en el análisis de la distribución de las conductas violentas y los roles del acoso.

Para comparar los indicadores estructurales (variables criterio), se empleó el rol como variable de agrupación a través de la prueba de Kruskall-Wallis para k muestras independientes, mientras que la comparación de tales indicadores de acuerdo al sexo se cumplió con la U de Mann-Whitney. Estos estadísticos no paramétricos se aplicaron en virtud que las variables analizadas no cumplieron los supuestos de normalidad y homocedasticidad exigidos para un procedimiento paramétrico. Los resultados aparecen descritos en la Tabla 3.

Tabla 3. Pruebas de comparación de diferencias de grupos para los indicadores estructurales de las redes según rol y sexo de los participantes.

\begin{tabular}{lllllll}
\hline \hline \multirow{2}{*}{ Indicador } & \multicolumn{2}{c}{ Descriptivos } & \multicolumn{2}{c}{ Kruskall Wallys $^{\mathrm{a}}$} & \multicolumn{2}{c}{ Mann-Whitney $^{\mathrm{b}}$} \\
\cline { 2 - 7 } & $\mathrm{M}$ & $\mathrm{DE}$ & $\mathrm{x}^{2}$ & $P$ & $\mathrm{U}$ & $P$ \\
\hline Grado nodal entrada & 80.21 & 53.09 & .39 & .941 & 11360 & .252 \\
Grado nodal salida & 77.60 & 20.99 & 1.56 & .667 & 11578 & .379 \\
Cercanía entrada & 79.21 & 14.04 & 1.54 & .673 & 12171.5 & .886 \\
Cercanía salida & 82.78 & 14.42 & 2.29 & .514 & 11474 & .313 \\
Intermediación & .66 & .48 & 6.02 & .110 & 11893.5 & .627 \\
\hline \hline
\end{tabular}

a Variable de agrupación: Rol. ${ }^{\mathrm{b}}$ Variable de agrupación: Sexo.

Los valores obtenidos en el análisis no ofrecieron diferencias significativas frente a las variables de agrupación seleccionadas por lo cual se asume la hipótesis de igualdad de las distribuciones de partida para los indicadores estructurales, es decir, los valores resultan independientes del rol y del sexo de los estudiantes implicados en conductas de acoso.

\section{Discusión}

El comportamiento de acoso entre pares es una de las problemáticas psicosociales que más interés genera en la actualidad dentro de los círculos educativos, constituyendo un asunto de necesaria participación de todos los actores de las comunidades académicas (estudiantes, docentes, directivos y familiares) de cara a su completa comprensión y necesaria intervención. En este estudio, se abordó el fenómeno del acoso entre iguales desde dos instancias complementarias, la primera de ellas centra- da en la identificación de las hostilidades más frecuentes dentro de un grupo de estudiantes, definiendo los roles asumidos y la interacción de estos con el sexo y el nivel de formación; y por otro lado, se analizó el papel de las redes de interacción dentro de la escuela en el desarrollo de los roles de acoso.

Frente a este horizonte de análisis, inicialmente se observó que la existencia de conductas hostiles entre pares es una realidad permanente, con índices elevados de aparición y con el desarrollo de una variedad de comportamientos agresivos que se dan indistintamente del género y del nivel formativo de los estudiantes. Nuestros resultados resaltan una elevada frecuencia de conductas centradas en las agresiones de tipo psicológico y verbal, así como expresiones sociales de violencia basadas en la ridiculización, y en menor medida, el uso de estrategias violentas de tipo físico de forma directa e indirecta. Estas características de las manifestaciones de acoso constituyen un patrón de comportamiento hostil entre iguales 
que corresponde con la evidencia ampliamente documentada en estudios previos (Ávila-Toscano et ál., 2010; Cepeda-Cuervo et ál., 2008; Del Barrio et ál., 2008; Garaigordobil \& Oñederra, 2008).

Del mismo modo, los roles asumidos por los participantes coinciden con evaluaciones anteriores en las que se destaca un número amplio de observadores (Del Barrio, Martin, Montero, Fernández \& Gutiérrez, 2001) y la participación significativa de un nutrido conjunto de actores reconocidos como agresores-víctimas (Ávila-Toscano et ál., 2012; Del Rey \& Ortega, 2008), mientras que el número de agresores exclusivos y de víctimas fue bastante similar. La distribución de estos roles no se asoció significativamente con el nivel de formación, pero sí con el sexo de los estudiantes, siendo notoriamente mayor las mujeres definidas como observadoras mientras que los varones se reportaron mayoritariamente involucrados con los demás roles. Sin embargo, es importante resaltar que el acoso entre iguales más que un asunto de género constituye una problemática en la que se ven involucrados todos los actores educativos, por lo que se requiere de su análisis desde perspectivas integrales con las cuales se propongan sistemas de intervención y prevención, dirigidos a la reducción del número de estudiantes involucrados en roles cuyas interacciones se basan en hostilidades.

El panorama de las características del maltrato identificado en este estudio refleja una participación amplia de estudiantes adoptando comportamientos violentos contras sus iguales, incluso, a diferencia de otros reportes (Ávila-Toscano et ál., 2010), se identificó un porcentaje alto de agresores que reconocen las conductas hostiles realizadas, mientras que las víctimas reportan en menor medida tales agresiones. Las respuestas a estas diferencias podrían hallarse en las redes sociales y en los patrones de interacción adoptados por los participantes, dado que, a diferencia de resultados ofrecidos por reportes previos (Estell, Farmer \& Cairns, 2007; Nansel et ál. 2001), las personas victimizadas en este estudio no carecen de núcleos de interacción y vinculación en sus escuelas.

Las víctimas, al igual que los agresores, conservan un margen de relaciones amplio, definido por numerosos contactos con los compañeros indistintamente del rol que estos asumen, de tal forma que las subredes estable- cidas por quienes son victimizados (es decir, su conjunto particular de relaciones dentro de la red general), se caracteriza por poseer densidad relacional, ubicándoles en posiciones centrales. Estas evidencias son reportadas por los similares niveles de indicadores estructurales hallados en cada conjunto de actores, los cuales no ofrecieron valores significativos para asumir la hipótesis de diferencias de los resultados expresados por cada indicador, de tal forma que el rendimiento general de las propiedades estructurales de la red resulta muy similar para cada uno de los estudiantes evaluados.

Para el caso de las víctimas, es posible asumir que la inclusión dentro de un sistema social en donde aparecen referenciadas como personas hacia las cuales existe reconocimiento y cercanía en los contactos, conlleve a la redefinición del significado de los comportamientos de acoso, los cuales podrían incluso ser omitidos en las relaciones conllevando al subregistro de conductas hostiles de las víctimas frente al reporte de los agresores; al sentirse integrado a un grupo, un individuo podría dejar de reconocer muestras de hostilidad siempre que las mismas formen parte del sistema de intercambios comunes de dicho grupo, tal y como es posible que las personas incluso puedan no identificar pautas de apoyo, cuando las mismas se dan naturalmente en las relaciones habituales de un conjunto de actores (Sagrestano, Feldman, Killingsworth, Woo \& Dunkel-Schetter, 1999).

En este sentido, resulta de suma prioridad el análisis de posibles expresiones de naturalización de la hostilidad en las relaciones entre iguales, en especial, porque este patrón de centralidad relacional de los actores implicados en el acoso se sostiene para el caso de quienes cumplen los roles de agresión. Nuestros resultados señalan en concordancia con evaluaciones anteriores (Kulig, Hall \& Kalischuk, 2008; Witvliet et ál., 2010), que los estudiantes que realizan conductas violentas contra sus pares conservan posiciones centrales dentro de las redes, más aún, los principales núcleos hallados en nuestro estudio con alta densidad en las relaciones, corresponde a agrupaciones conformadas por la interacción intensa entre conjuntos numerosos de agresores-víctimas y pocas víctimas, datos que coinciden con lo reportado por Farmer et ál. (2010), mientras que los agresores exclusivos se encuentran en menor medida dentro de tales relaciones. 
Desde finales de la primera década del presente siglo, autores como Del Rey y Ortega (2008), han señalado la necesidad de centrar la atención en el papel que cumplen los actores con el rol mixto de agresor-víctima por las implicaciones psicoemocionales que dicho rol representa, y las evidencias posteriores -como las reseñadas en este estudio- recalcan la importancia de reconocer todas las particularidades asociadas con este papel en las relaciones de acoso, en especial porque a todas luces, dentro de nuestras evidencias representan el conjunto de actores de mayor participación, control, densidad y posición social en las redes de acoso.

$\mathrm{Al}$ asumir este rol mixto, los estudiantes son objeto de relaciones agresivas y al mismo tiempo dirigen sus hostilidades hacia los demás, sin embargo, también resultan relacionalmente apetecibles para establecer vinculaciones ajenas al ejercicio agresivo, de hecho, les permite sobresalir como actores con un nivel amplio de control social, de forma que pese a desarrollar comportamientos hostiles son identificados por sus compañeros como fuentes de interacción deseable.

Autores como Ojala y Nesdale (2004), han señalado que la forma particular en que un integrante de un grupo se comporta está asociado directamente con el sistema de interacción aprobado y reconocido por dicho grupo, de tal forma que las expresiones agresivas existentes entre iguales pueden ser reconocidas como mecanismos válidos de interacción, patrón que es asumido incluso por las víctimas, quienes no serían marginadas dado que actuarían dentro de un escenario social en el que la agresividad es un medio de comunicación y de vinculación. Esto conduce a pensar en un posible estado de naturalización del conflicto al que pueden llegar los grupos de pares por lo cual no se distinguirían mayores diferencias en cuanto a los indicadores de sus redes, si bien es esta una hipótesis que requiere de la intervención de otras variables tales como la significación otorgada al acoso, la percepción de las relaciones e incluso, la exploración de los mecanismos de apoyo de las redes.

Ahora bien, los hallazgos de este estudio reconocen la existencia de ciertas limitaciones tales como la naturaleza de la muestra, dado que si bien se trató de un grupo naturalmente constituido, el mismo obedeció a estudiantes con características muy similares, mientras que en estudios previos, en los que se han identificado diferencias en las redes de cada actor, los participantes son notoria- mente distintos por características como la etnicidad y los acervos culturales a los que pertenecen, variables que con frecuencia median los resultados en los fenómenos sociales. Por otra parte, en el procedimiento formulado no se incluyen mecanismos de apoyo social dentro del análisis de las redes, condición que podría ofrecer resultados diversos respecto al nivel de acceso a recursos de ayuda de acuerdo al rol.

Ahora bien, pese a estas limitaciones, los datos reseñados por nuestro estudio representan un aporte tanto en el reconocimiento de los sistemas de interacción entre pares inmersos en episodios de acoso, como también la posibilidad de plantearse cuestionamientos acerca de si realmente las diferencias en el sistema relacional están dadas por los roles asumidos o por la participación del sexo de los estudiantes. Desde nuestra óptica, y dada la ausencia de diferencias significativas de los indicadores de las redes frente al sexo y al rol, consideramos que el acoso escolar no necesariamente es problema de géneros, ni las redes establecidas dependen necesariamente de esta variable, en cambio, la exploración detallada de las estructuras de relación, los mecanismos de vinculación, la significación otorgada a las relaciones y los beneficios sociales derivados de la participación en el acoso, constituyen fuentes esenciales de análisis que podrían ofrecer propuestas válidas de intervención en pro de la reducción y prevención de esta problemática.

Adicionalmente, no debe dejarse de lado la participación de las redes de interacción sobre los efectos que a mediano o largo plazo pueden ejercer las conductas de acoso sobre el estado psicológico de los implicados. Las evidencias reportadas en este estudio abren una discusión importante acerca de la participación de variables como la estructura de las redes, el comportamiento de sus indicadores (e incluso la participación de mecanismos de soporte) en el desarrollo o atenuación de los efectos del maltrato entre iguales. En este estudio en particular, se responde, al menos al nivel más inicial del análisis de dicho fenómeno, dado que el enfoque estructural conlleva a generar cuestionamientos sobre la participación de condiciones relacionales que inciden en una problemática hasta ahora enfocada desde lo atributivo; en esta línea de análisis puede haber incluso un importante acervo de estudios con implicaciones valiosas sobre la salud emocional y la adaptación psicológica de los menores envueltos en conductas de acoso. 


\section{Referencias}

Arseneault, L., Bowes, L. \& Shakoor, S. (2010). Bullying Victimization in Youths and Mental Health Problems: 'Much Ado About Nothing'? Psychological Medicine, 40(5), 717-729.

Ávila-Toscano, J. H. (2009). Redes sociales, generación de apoyo social ante la pobreza y calidad de vida. Revista Iberoamericana de Psicología: Ciencia y Tecnología, 2 (2), 65-73.

Ávila-Toscano, J. H., Gutiérrez, B. \& Pérez, J. (2011). Indicadores estructurales y conglomerados de actores en la red social de una subcultura urbana. Revista Colombiana de Psicología, 20 (2), 193-207.

Ávila-Toscano, J. H. \& Madariaga, C. (2012). Redes sociales: un ejercicio caracterológico. En: J. H., ÁvilaToscano. Redes sociales y análisis de redes. Aplicaciones en el contexto comunitario y virtual. (pp. 16-47). Barranquilla: Corporación Universitaria Reformada.

Ávila-Toscano, J. H., Osorio, L., Cuello, K., Cogollo, N. \& Hernández, K. (2010). Conducta bullying y su relación con la edad, sexo y nivel de formación. Revista Psicogente, 13 (23), 13-26.

Avilés, J. M. \& Monjas, I. (2005). Estudio de incidencia de la intimidación y el maltrato entre iguales en la educación secundaria obligatoria mediante el cuestionario CIMEI (Avilés, 1999) -Cuestionario sobre intimidación y maltrato entre iguales-. Anales de Psicología, 21 (1), 27-41.

Bidart, C. \& Degenne, A. (2005). Introduction: the Dynamics of Personal Networks (Editorial). Social Networks, 27(4), 283-287.

Bloom, J. R. \& Kessler, L. (1994). Emotional Support Following Cancer: a Test of the Stigma and Social Activity Hypotheses. Journal of Health and Social Behavior, 35(2), 118-133.

Boivin M. (2005). The Origin of Peer Relationship Difficulties in Early Childhood and their Impact on Children's Psychosocial Adjustment and Development. En: R. E. Tremblay, R. G. Barr \& R. D. Peters (Eds). (pp. 1-7). Encyclopedia on Early Childhood Development. Montreal, Quebec: Centre of Excellence for Early Childhood Development. Disponible en: www.child-encyclopedia.com/documents/BoivinANGxp.pdf.
Borgatti, S. P., Everett, M. G. \& Freeman, L. C. (2002). Ucinet for Windows: Software for Social Network Analysis. Harvard, MA: Analytic Technologies.

Borgatti, S. P. (2002). Netdraw Network Visualization. Harvard, MA: Analytic Technologies.

Carter, W. C. \& Feld, S. L. (2004). Principles Relating Social Regard to Size and Density of Personal Networks, with Applications to Stigma. Social Networks, 26 (4), 323-329.

Cepeda-Cuervo, E., Pacheco-Durán, P., García-Barco, L. \& Piraquive-Peña, C. (2008). Acoso escolar a estudiantes de educación básica y media. Revista Salud Pública, 10 (4), 517-528.

Cerezo, F. \& Ato, M. (2010). Social Status, Gender, Classroom Climate and Bullying Among Adolescents Pupils. Anales de Psicología, 26 (1), 137-144.

Defensor del Pueblo. (2007). Violencia escolar: el maltrato entre iguales en la Educación Secundaria Obligatoria 1999-2006. Serie: Informes, estudios y documentos. Madrid: España.

Del Barrio, C., Martín, E., Montero, I., Gutiérrez, H., Barrios, A. \& de Dios, M. J. (2008). Bullying and social exclusion in Spanish secondary schools: $\mathrm{Na}-$ tional trends from 1999 to 2006. International Journal of Clinical and Health Psychology, 8 (3), 657-677.

Del Rey, S. \& Ortega, R. (2008). Bullying en los países pobres: prevalencia y coexistencia con otras formas de violencia. Internacional Journal of Psychology and Psychological Therapy, 8 (1), 39-50.

Del Barrio, C., Martín, E., Montero I., Fernández, I. \& Gutiérrez, H. (2001). Peer Bullying in Spanish Secondary Schools: A National Scale Study for the Ombudsman's Report on School Violence. In M. Martínez (Ed.). Prevention and Control of Aggression and the Impact on its Victims (pp. 155-160). New York: Kluwer Academic/Plenum.

Estell, D., Farmer, T. \& Cairns, B. (2007). Bullies and Victims in Rural African American Youth: Behavioral Characteristics and Social Network Placement. Aggressive Behavior, 33 (2), 145-159.

Farmer, T., Petrin, R., Robertson, D., Fraser, M., Hall, C., Day, S. \& Dadisman, K. (2010). Peer Relations of Bullies, Bully-Victims, and Victims: The Two Social Worlds of Bullying in Second-Grade Classrooms. The Elementary School Journal, 110 (3), 364-392. 
Garaigordobil, M. \& Oñederra, J. A. (2008). Bullying: Incidente of Peer Violence in the Schools of the Autonomus Community of the Basque Country. International Journal of Psychology and Psychological Therapy, 8 (1), 51-62.

Gini, G. \& Pozzoli, T. (2009). Association Between Bullying and Psychosomatic Problems: A Meta-Analysis. Pediatrics, 123 (3), 1059-1065.

Gifford-Smith, M. \& Brownell, C. (2003). Childhood Peer Relationships: Social Acceptance, Friendships, and Peer Networks. Journal of School Psychology, 41, 235-284.

Hawe, P., Webster, C. \& Shiell, A. (2004). A Glossary of Terms for Navigating the Field of Social Network Analysis. Journal Epidemiology Community Health, 58, 971-975.

Hinduja, S. \& Patchin, J. W. (2010): Bullying, Cyberbullying, and Suicide. Archives of Suicide Research, 14 (3), 206-221.

Hoyos, O., Aparicio, J., Heilbron, K. \& Schamun. (2004). Representaciones sobre el maltrato entre iguales en niñas y niños escolarizados de 9, 11 y 13 años de nivel socioeconómico alto y bajo de la ciudad de Barranquilla. Psicología desde el Caribe, 14, 150-172.

Kalish, Y. \& Robins, G. (2006). Psychological Predispositions and Network Structure: The Relationship Between Individual Predispositions, Structural Holes and Network Closure. Social Networks, 28 (1), 56-84.

Kulig, J. C., Hall, B. L. \& Kalischuk, R. G. (2008). Bullying Perspectives Among Rural Youth: a Mixed Methods Approach. Rural and Remote Health, 8 (2), 923-933.

Madariaga, C., Gallardo, L. D., Salas, F. \& Santamaría, E. (2001). Violencia política y sus efectos en la identidad psicosocial de los niños desplazados. El caso de La Cangrejera. Psicología desde el Caribe, 10, 88-106.

Merino, J. (2008). El acoso escolar-bullying una propuesta de estudio desde el Análisis de Redes Sociales (ARS). Revista d'estudis de la Violencia, 4, 1-17.

Montero, I. \& León, O. (2007). A Guide for Naming Research Studies in Psychology. International Journal of Clinical and Health Psychology, 7 (3), 847-862.

Nansel, T. R, Overpeck, M., Pilla, R. S., Ruan, W. J., Simons-Morton, B. \& Scheidt, P. (2001). Bullying Behaviors Among us Youth Prevalence and Association with Psychosocial Adjustment. Journal American Medical Assotitation, 285 (16), 2094-2100.
Ojala, K. \& Nesdale, E. (2004). Bullying and Social Identity: The Effects of Group Norms and Distinctiveness Threat on Attitudes Towards Bullying. British Journal of Developmental Psychology, 22 (1), 19-35.

Ortega, R. (2002). Lo mejor y lo pero en las redes de iguales: juego, conflicto y violencia. Revista Interuniversitaria de Formación del Profesorado, 44, 93-13.

Plata, C., Riveros, M. \& Moreno, J. (2010). Autoestima y empatía en adolescentes observadores, agresores y víctimas del bullying en un colegio del municipio de Chía. Psychologia: avances de la disciplina, 4 (2), 99-112.

Riaño, E. J. (2008). Descripción de un modo de propagación de relaciones violentas en una muestra de niños y niñas entre 9 y 14 años. Tesis Psicológica, 3, 70-85.

Sagrestano, L., Feldman, P., Killingsworth, C. H., Woo, G. \& Dunkel-Schetter, C. H. (1999). Ethnicity and Social Support During Pregnancy. American Journal of Community Psychology, 27 (6), 869-898.

Samper, M. (2004). Redes sociales y comunicación entre experimentadores campesinos en Puriscal, Costa Rica. Revista de Ciencias Sociales (Cr), 4 (106), 143163.

Takács, K. (2007). Effects of Network Segregation in Intergroup Conflict: An Experimental Analysis. Connections, 27 (2), 59-76.

Trujillo, H., Mañas, F. \& González-Cabrera, J. (2010). Evaluación de la potencia explicativa de los grafos de redes sociales clandestinas con UciNet y NetDraw. Universitas Psychologica, 9 (1), 67-78.

Witvliet, M., Olthof, T., Hoeksma, J., Goossens, F., Smits, M. \& Koot, H. (2010). Peer Group Affiliation of Children: The Role of Perceived Popularity, Likeability, and Behavioral Similarity in Bullying. Social Development, 19 (2), 285-303.

Xie, H., Farmer, T. W. \& Cairns, B. D. (2003). Different Forms of Aggression Among Inner-City AfricanAmerican Children: Gender, Configurations, and School Social Networks. Journal of School Psychology, 41 (5), 355-375.

Zabaraín, S. \& Sánchez, D. (2009). Implicaciones del bullying o maltrato entre pares en el desarrollo psicoafectivo de niños y niñas en etapa de latencia. Psicogente, 12 (22), 407-421. 